\title{
Fluorescence studies on radiation oxidative damage to membranes with implications to cellular radiosensitivity
}

\author{
K P MISHRA \\ Radiation Biology Division, Bhabha Atomic Research Centre, \\ Mumbai 400 085, India \\ e-mail:kpm@magnum.barc.ernet.in; mishra_kaushala@rediffmail.com
}

\begin{abstract}
Radiation oxidative damage to plasma membrane and its consequences to cellular radiosensitivity have received increasing attention in the past few years. This review gives a brief account of radiation oxidative damage in model and cellular membranes with particular emphasis on results from our laboratory. Fluorescence and ESR spin probes have been employed to investigate the structural and functional alterations in membranes after $\gamma$-irradiation. Changes in the lipid bilayer in irradiated unilamellar liposomes prepared from egg yolk lecithin (EYL) were measured by using diphenylhexatriene (DPH) as a probe. The observed increase in DPH polarization and decrease in fluorescence intensity after $\gamma$-irradiation of liposomes imply radiationinduced decrease in bilayer fluidity. Inclusion of cholesterol in liposome was found to protect lipids against radiation damage, possibly by modulation of bilayer organization e.g. lipid packing. Measurements on dipalmitoyl phosphatidylcholine (DPPC) liposomes loaded with 6-carboxyfluorescein $(\mathrm{CF})$ showed radiation dose-dependent release of the probe indicating radiation-induced increased permeability. Changes in plasma membrane permeability of thymocytes were monitored by fluorescein diacetate (FDA) and induced intracellular reactive oxygen species (ROS) were determined by 2,7-dichlorodihydro fluorescein diacetate (DCH-FDA). Results suggest a correlation between ROS generation and membrane permeability changes induced by radiation within therapeutic doses $(0-10 \mathrm{~Gy})$. It is concluded that increase in membrane permeability was the result of ROS-mediated oxidative reactions, which might trigger processes leading to apoptotic cell death after radiation exposure.
\end{abstract}

Keywords. Membrane oxidative damage; cellular radiosensitivity; DPH fluorescence; lipid peroxidation; liposomal membrane; thymocyte membrane permeability.

\section{Introduction}

Radiation damage to cells and tissues involves generation of reactive oxygen species (ROS) followed by alterations in lipids, DNA and proteins, which eventually lead to cellular dysfunction or death. Studies on cellular radiosensitivity have often been devoted to evaluating DNA damage and repair. However, response of cells to radiation does not fully account in terms of efficiency of DNA repair. It has long been held that membranes constitute another important cellular target for radiation action including a site for oxygen effect. ${ }^{1}$ However, these suggestions were advanced when information on structure and components of cellular membrane was limited. In recent years, considerable evidence has accumulated suggesting involvement of membrane damage in development of radiationinduced cell injury and eventually in cell death. ${ }^{2,3}$ However, molecular details of oxidative damage to membranes and its role in the mechanisms of cell death are lacking. Research has shown that radiation-generated free radicals are involved in initiating 
oxidative damage to vital cellular components, which are ultimately manifested in pathogenesis including induction of cancer. ${ }^{4,5}$ The damaging events at the molecular level, such as peroxidation of lipids, are believed to cause functional and structural changes in membrane. ${ }^{6-8}$ Radiation-generated ROS are also shown to cause membrane peroxidative damage and to modulate cytosolic signaling cascade, which regulate processes leading to apoptotic cell death. Liposomes offer a suitable model for membrane to investigate the mechanism of radiation-induced damage because the composition of liposomes can be controlled and modified by utilizing appropriate lipids. Thymocytes have been employed as a sensitive cell system for radiation action. Fluorescence probe techniques offer powerful and sensitive tool for quantitative evaluation of radiation-induced generation of ROS and oxidative membrane damage, which has been employed to determine changes in permeability and fluid properties of membrane after radiation exposure. Several other spectroscopic techniques such as NMR, ESR, IR and fluorescence are witnessing rapid advancements with the promise for unravelling membrane dynamics and molecular characterization of membrane-specific radiation oxidative damage. ${ }^{9,10} \mathrm{DPH}$ fluorescence polarization has been shown to monitor radiation-induced structural and organizational changes in cell membrane $e^{9,11,12}$ because of its high extinction coefficient and preferential high localization in membrane bilayer. FDA has commonly been employed for determination of cell viability and occurrence of apoptosis together with fluorescent dyes, such as, propidium iodide/ethidium bromide. FDA freely enters into cells and is hydrolysed by cytoplasmic esterases to fluorescein. Cells with intact membrane retain fluorescein due to its polarity but change in membrane permeability allows leakage of fluorescein from the cell, reflected in decrease of fluorescence intensity in cells. DCHFDA, a derivative of FDA, has been used to determine intracellular reactive oxygen species (figure 1a and $\mathrm{b}$ ). From the studies on radiation oxidative damage to liposomes and thymocytes using appropriate fluorescence probes, it has been shown that permeability and fluidity of membrane are remarkably altered after irradiation, which varied with the radiation dose. Presence of structure modulating agents (e.g. cholesterol) or antioxidant molecules (e.g. tocopherol, ascorbic acid) in the membrane protected lipids against radiation, suggesting involvement of free radicals in the damage process. There seems to exist a relationship between generated ROS and permeability changes after

(a)<smiles>CC(=O)Oc1ccc2c(c1)Oc1cc(OC(C)=O)ccc1C21OC(=O)c2ccccc21</smiles>

(b)

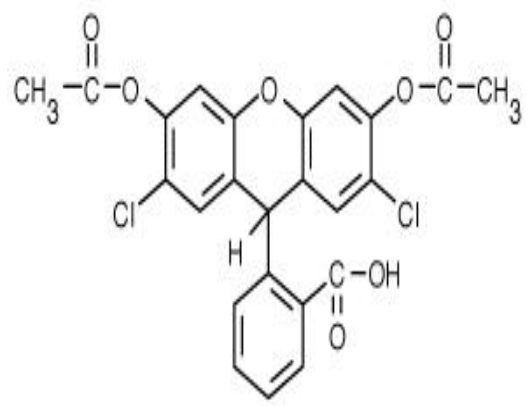

Figure 1. Chemical structure of fluorescence probes employed for determination of membrane permeability, FDA (a) and reactive oxygen species, DCH-FDA (b). 
radiation exposure. These observations have important implications in the mechanism of apoptotic cell death and cellular radiosensitivity.

\section{Materials and methods}

\subsection{Chemicals}

DPPC, EYL, thiobarbituric acid (TBA) and DPH were obtained from Sigma. CF was obtained from Eastman Kodak and purified. ${ }^{13}$ DCH-FDA was purchased from Molecular Probes, USA.

\subsection{Preparation and irradiation of liposomes}

Egg yolk lecithin and DPPC liposomes were prepared by following protocol reported earlier. ${ }^{11,12}$ Unilamellar liposomal suspensions of egg yolk lecithin or DPPC were irradiated by ${ }^{60} \mathrm{Co} \gamma$-rays after appropriate dilutions (dose rate: $11 \mathrm{~Gy} / \mathrm{min}$ ).

\subsection{Measurement of permeability and fluidity in irradiated liposomes}

Permeability of irradiated DPPC vesicles was monitored by leakage of pre-loaded CF after irradiation, followed by incubation at $25^{\circ} \mathrm{C}$. CF fluorescence was measured with a spectrofluorimeter $\left(\lambda_{\mathrm{ex}}=490 \mathrm{~nm}\right.$ and $\left.\lambda_{\mathrm{em}}=520 \mathrm{~nm}\right)$ LS50B, Perkin-Elmer, USA. Concentration of $\mathrm{CF}$ in liposomes $(\sim 100 \mathrm{mM})$ was chosen such that it gave practically no fluorescence due to self-quenching. However, after irradiation, leakage of CF occurred giving intense fluorescence due to dilution in the external aqueous medium (figure 2). Permeability of liposomes was expressed in terms of relative fluorescence intensity, which was the ratio of fluorescence between a particular liposome sample and the total fluorescence obtained by disruption of liposomes with Triton X-100 (0.01\%) and multiplied by 100 .

Radiation-induced membrane oxidative damage of EYL liposomes was measured after labelling with $10 \mu \mathrm{M}$ DPH in PBS at $25^{\circ} \mathrm{C}$ (lipid/probe molar ratio 100). DPH fluorescence and polarization in liposomes were measured in PBS at $25^{\circ} \mathrm{C}\left(\lambda_{e x}=357 \mathrm{~nm}\right.$ and $\lambda_{\mathrm{em}}=425 \mathrm{~nm}$ ). The fluorescence from liposomes without the probe was always less than $1 \%$ as compared to the labelled liposomes.

\subsection{Lipid peroxidation in EYL liposomes}

The radiation-induced peroxidation in liposomal samples was measured spectrophotometrically by MDA formation following TBA method described elsewhere. ${ }^{11}$

\subsection{Preparation of thymocyte suspension and labelling by DCFH-DA}

Thymocytes cell suspensions were prepared in phosphate buffer saline from 4-6 weeks old mice as described earlier. ${ }^{14}$ Thymocytes $\left(1 \times 10^{7}\right.$ cells $\left./ \mathrm{ml}\right)$ were labelled with DCFHDA in PBS by mixing in a stock solution of DCFH-DA $(6.3 \mathrm{mg} / \mathrm{ml})$ in ethanol (final dilution: 1000 times in cell suspension). Incubation was carried out at $25^{\circ} \mathrm{C}$ for $20 \mathrm{~min}$. Labelled thymocytes were irradiated $(10 \mathrm{~Gy}$, dose rate: $0.6 \mathrm{~Gy} / \mathrm{min})$ at $25^{\circ} \mathrm{C}$ by ${ }^{60} \mathrm{Co} \gamma$ rays. Fluorescent cells were scored under fluorescence microscope and labelled cell fluorescence was measured fluorimetrically $\left(\lambda_{\mathrm{ex}}=490 \mathrm{~nm}, \lambda_{\mathrm{em}}=520 \mathrm{~nm}\right)$. 


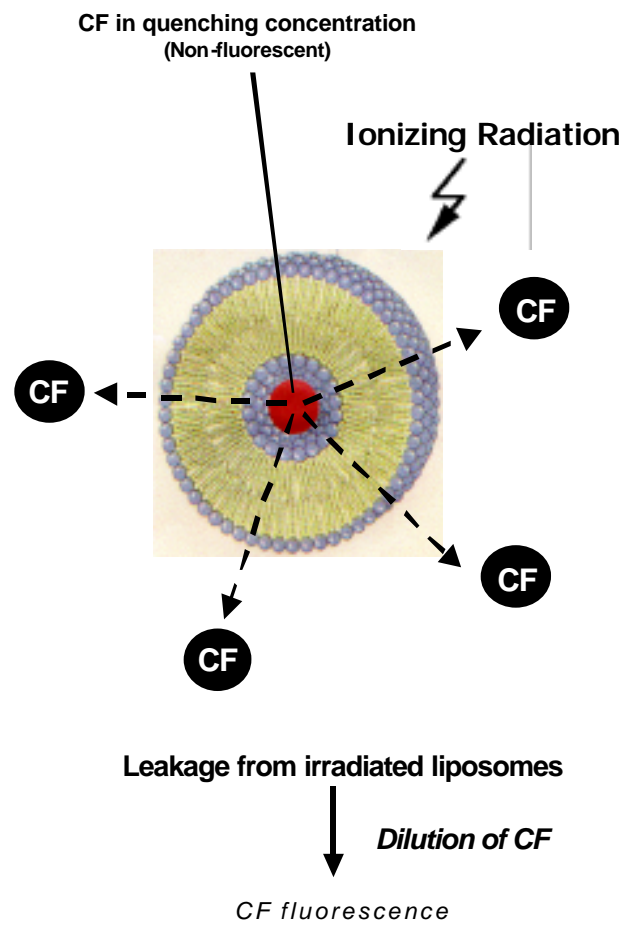

Figure 2. Schematic representation of measurement of radiation-induced liposomal membrane permeability by $\mathrm{CF}$.

\section{Results and discussion}

\subsection{Modification of radiation oxidative damage in liposomal membrane}

Radiation effects on membrane constitute an important study in radiobiology because damage to cellular membranes has been found contributing to development of radiation cell injury. ${ }^{2,6,7,14}$ The factors that modify membrane damage show considerable modification to cellular radiosensitivity. It is generally believed that radiation membrane oxidative damage is mediated through radiation-generated ROS, which react with membrane components causing disruption of membrane structure. Studies in our laboratory on membrane oxidative damage after irradiation of unilamellar liposomes by DPH fluorescence have shown radiation dose dependent decrease in fluidity and increase in MDA formation, suggesting involvement of lipid peroxidation mechanism in modification of bilayer structure (figure 3). Inclusion of cholesterol in bilayer composition produced concentration-dependent protection of bilayer lipids against radiation. ${ }^{11}$ Results have also demonstrated that irradiation of liposomes incorporating antioxidants exhibited substantial protection from radiation damage. ${ }^{8,12}$ Increasing concentration of cholesterol $(0-$ 50 mole $\%$ ) in liposome composition showed a linear increase in membrane microviscosity as measured by DPH polarization. It was interesting to find that MDA formation and DPH polarization in irradiated liposomal membrane as a function of radiation dose 


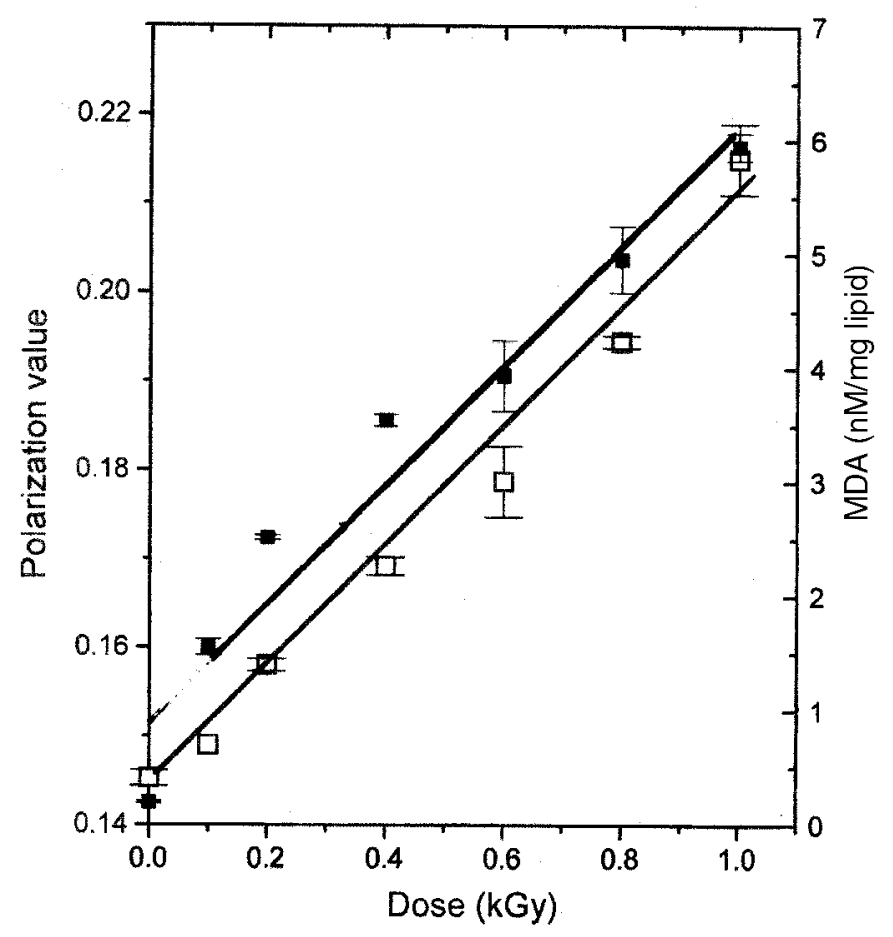

Figure 3. MDA formation and DPH polarization in EYL unilamellar liposomes. Sonicated liposomes were prepared with egg yolk lecithin followed by irradiation at different doses $(0 \cdot 1-1 \mathrm{kGy})$. MDA ( $\square$ ) and DPH polarization ( $\mathbf{\square})$ in control and irradiated liposomes were measured as described in $\S 2$.

showed a linear relationship (figure 3) suggesting suitability of polarization method for determination of structural alterations due to radiation-induced lipid peroxidation. In addition, it was demonstrated that radiosensitivity of membrane was modified by modulation of membrane structure ${ }^{11}$ or by the presence of ROS scavengers like o-tocopherol. ${ }^{8}$ These results on model membrane system need to be extended to studies on cells. It would be interesting to verify by further experiments if enrichment of cells by cholesterol confers resistance to radiation effects on them.

\subsection{Radiation-induced permeability changes in DPPC vesicles}

Changes in membrane permeability in irradiated DPPC liposomes in the presence of lipophilic and hydrophilic antioxidants were studied by monitoring the leakage of preloaded CF. ${ }^{12}$ Irradiation of DPPC liposomes yielded radiation dose-dependent increase in $\mathrm{CF}$ fluorescence, which showed a biphasic response up to $6 \mathrm{kGy}$. Percent release of CF from vesicles irradiated to a dose of $0.7 \mathrm{kGy}$ was significantly higher compared to control which was found to decrease at higher radiation and the leakage of CF was insignificantly small from the liposomes after irradiation to a dose of $6 \mathrm{kGy}$ up to $7 \mathrm{~h}$ incubation. ${ }^{12}$ These results have been interpreted in terms of induced fluidization of lipid bilayer up to 
certain doses followed by rigidization to increasing dose of $\gamma$-radiation indicative of a complex mechanism of oxidative damage. Moreover, it was found that liposomes irradiated with increasing dose rates showed a decreased CF leakage, suggesting an inverse dose rate effect. This observation shows involvement of free radical chain mechanism in the damage process, which is a commonly observed phenomenon in radiation lipid damage. ${ }^{6}$ It was further found that presence of antioxidants in the vesicles during irradiation remarkably prevented release of $\mathrm{CF}$, suggesting protection to radio-oxidative damage. A comparison of protective effects of antioxidants showed that $\alpha$ tocopherol offered more effective protection than ascorbic acid against radiation damage.

\subsection{Radiation-induced permeability changes in mouse thymocytes}

Measurement of DCFH-DA fluorescence after incorporation in thymocytes as a function of radiation dose (0-10 Gy) showed a dose-dependent increased probe fluorescence, suggesting generation of ROS with increasing radiation dose (this lab, unpublished data), which was, however, significantly modified by the presence of intracellular antioxidants. Irradiation of thymocytes loaded with DCFH-DA showed intense fluorescence as observed under fluorescence microscope and the proportion of fluorescing cells followed radiation dose (figure 4). Based on these results and those obtained from ESR spin probe measurements, it is suggested that measurement of radiation-induced permeability may offer a potential indicator/dosimeter of radiation exposure. ${ }^{15}$

The oxidative damage of membrane may be triggered by radiation-generated ROS reflected in structural and functional alterations which may affect membrane-associated or intracellular cell signal cascade eventually resulting in cell death especially apoptotic death. ${ }^{16,17}$ It is suggested that damage to membrane may be a critical target for radiation

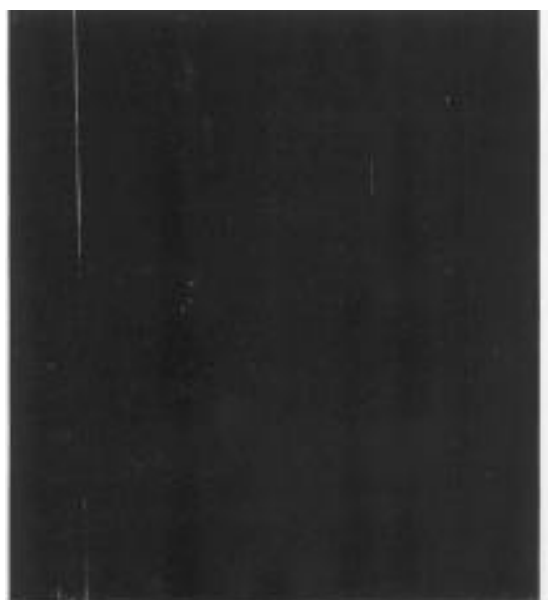

Control

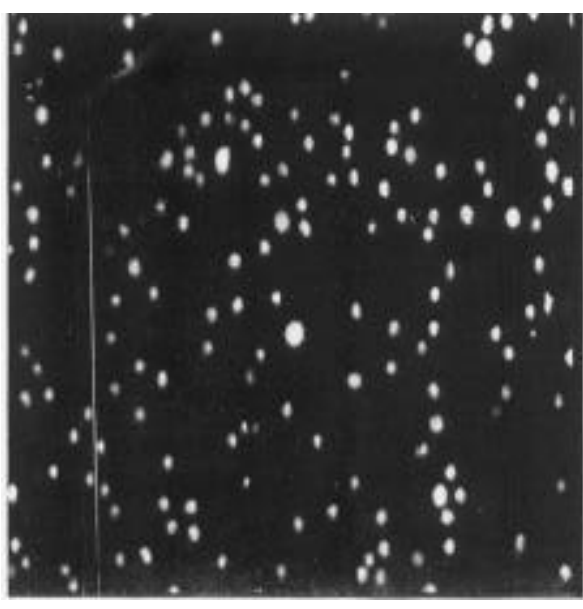

Irradiated (10 Gy)

Figure 4. Reactive oxygen species (ROS) in thymocytes after radiation exposure using DCH-FDA as fluorescence probe. Mouse thymocytes were labelled with DCHFDA before irradiation. The cells were examined under fluorescence microscope with and without labelling. 
action and more data need to be accumulated to establish if factors modifying membrane function can also modify cell function. A wealth of new information has accumulated over the years but advanced biophysical techniques and molecular biological tools are expected to provide a deeper and better understanding of the processes involved in radiation cellular sensitivity.

\section{Conclusions}

It has been demonstrated that membrane oxidative damage was initiated by radiationinduced ROS which could be modified by structure modulating factors such as cholesterol or membrane-specific antioxidants e.g. tocopherol. Post-irradiation permeability changes in thymocyte membrane suggest propagation of initial events with the passage of time. Results have shown that fluorescence probes give a good account of chemical and structural alterations in membranes. Furthermore, it is suggested that measurement of membrane injury may provide an indicator of radiation exposure. It is speculated that membrane damage may initiate or contribute to processes leading to induction of apoptotic cell death. Studies on membrane oxidative damage may form a basis for cellular radiosensitivity, which have considerable implications in basic radiobiology and in clinical cancer radiotherapy.

\section{References}

1. Alper T 1997 The role of membrane damage in radiation-induced cell death. In Membrane toxicity (eds) M W Miller and A E Shamou (New York: Plenum) p. 139

2. Ramakrishnan N, McClain D E and Catravas G N 1993 Int. J. Radiat. Biol. 63693

3. Haimovitz-Friedmann A, Kan C C, Ehleiter D, Persaud R C, McLoughlin M, Fucks Z and Kolesnick R N 1994 J. Exp. Med. 180525

4. Halliwell B and Gutteridge J M C 1989 Free radicals in biology and medicine 2nd edn (Oxford: University Press)

5. Bandyopadhyay U, Das D and Banerjee R K 1999 Curr. Sci. 77658

6. Stark G 1991 Biochim. Biophys. Acta 1071103

7. Pandey B N and Mishra K P 1999 Adv. Radiat. Biol. Peace, UPZS (Suppl.) 245

8. Pandey B N and Mishra K P 2002 J. Biochem. Mol. Biol. Biophys. 6267

9. McElhaney R N 1994 Techniques for measuring lipid phase state and fluidity in biological membranes. In Temperature adaptation of biological membranes (ed.) A R Cossins (London: Portland) p. 31

10. Pandey B N and Mishra K P 2000 Appl. Magn. Reson. 18483

11. Pandey B N and Mishra K P 1999 Radiat. Phys. Chem. 54481

12. Marathe D and Mishra K P 2002 Radiat. Res. 157685

13. Pandey B N and Mishra K P 1997 J. Surf. Sci. Technol. 13113

14. Pandey B N and Mishra K P 2002 Int. J. Low Radiat. 1 (in press)

15. Mishra K P 2002 Adv. ESR Appl. 18243

16. Pandey B N and Mishra K P 1999 Res. Bull. Panjab Univ. 49175

17. Pandey B N and Mishra K P 2001 Iran. J. Med. Sci. (in press) 